\title{
Noninformative priors for the log-logistic distribution
}

\author{
Sang Gil Kang ${ }^{1} \cdot$ Dal Ho Kim² ${ }^{2}$ Woo Dong Lee ${ }^{3}$ \\ ${ }^{1}$ Department of Computer and Data Information, Sangji University \\ ${ }^{2}$ Department of Statistics, Kyungpook National University \\ ${ }^{3}$ Department of Asset Management, Daegu Haany University \\ Received 15 October 2013, revised 16 December 2013, accepted 3 January 2014
}

\begin{abstract}
In this paper, we develop the noninformative priors for the scale parameter and the shape parameter in the log-logistic distribution. We developed the first and second order matching priors. It turns out that the second order matching prior matches the alternative coverage probabilities, and is a highest posterior density matching prior. Also we revealed that the derived reference prior is the second order matching prior for both parameters, but Jeffrey's prior is not a second order matching prior. We showed that the proposed reference prior matches the target coverage probabilities in a frequentist sense through simulation study, and an example based on real data is given.
\end{abstract}

Keywords: Log-logistic distribution, matching prior, reference prior.

\section{Introduction}

The log-logistic distribution is well known in survival analysis of data sets such as survival times of cancer patients in which the hazard increases initially and decreases later (Bennett, 1983). Also in economic studies of distributions of wealth or income, it is known as Fisk distribution (Fisk, 1961) and is considered as an equivalent alternative to a log-normal distribution. For further details on the importance and applications of a log-logistic distribution one may refer to Shoukri et al. (1988), Geskus (2001), Robson and Reed (1999) and Ahmad et al. (1988).

In this paper, we focus on the development of noninformative priors in the log-logistic distribution. There are two different notions of noninformative priors. One is a probability matching prior introduced by Welch and Peers (1963) which matches the posterior and frequentist probabilities of confidence intervals. Interest in such priors has been revived with the work of Stein (1985) and Tibshirani (1989). Among others, we may cite the work of Mukerjee and Dey (1993), DiCiccio and Stern (1994), Datta and Ghosh (1995, 1996), Mukerjee and Ghosh (1997). Kang et al. (2012, 2013a, 2013b) developed the probability matching priors for the several distributions.

\footnotetext{
1 Professor, Department of Computer and Data Information, Sangji University, Wonju 220-702, Korea.

2 Professor, Department of Statistics, Kyungpook National University, Daegu 702-701, Korea.

3 Corresponding author: Professor, Department of Asset Management, Daegu Haany University, Kyungsan 712-715, Korea. E-mail: wdlee@dhu.ac.kr
} 
The other is the reference prior introduced by Bernardo (1979) which maximizes the Kullback-Leibler divergence between the prior and the posterior. Ghosh and Mukerjee (1992), and Berger and Bernardo $(1989,1992)$ give a general algorithm to derive a reference prior by splitting the parameters into several groups according to their order of inferential importance. This approach is very successful in various practical problems (Kang, 2011; Kim et al. 2009). Quite often reference priors satisfy the matching criterion described earlier.

The outline of the remaining sections is as follows. In Section 2, we develop first order and second order probability matching priors. We reveal that the second order matching prior is a highest posterior density (HPD) matching prior and matches the alternative coverage probabilities up to the second order. Also we derive the reference priors for the parameter. It turns out that the reference prior and Jeffreys' prior are the second order matching prior. Section 3 devotes to show that the propriety of the posterior distribution for the general prior including the reference prior and the matching prior. In Section 4, simulated frequentist coverage probabilities under the proposed prior and an example are given.

\section{The noninformative priors}

Suppose that $X$ is distributed random variable according to the log-logistic distribution $\mathcal{L} \mathcal{L}(\alpha, \beta)$ with the scale parameter $\alpha$ and the shape parameter $\beta$. Then the probability density function of log-logistic distribution of $X$ is given by

$$
f(x \mid \alpha, \beta)=\frac{\beta \alpha^{\beta} x^{\beta-1}}{\left(\alpha^{\beta}+x^{\beta}\right)^{2}}, x \geq 0, \alpha>0, \beta>0 .
$$

We want to make a Bayesian inference about the scale parameter and the shape parameter based on noninformative prior or objective priors. Therefore we will develop the noninformative priors for both parameters of log-logistic distribution.

\subsection{The probability matching priors}

For a prior $\pi$, let $\theta_{1}^{1-\alpha}(\pi ; \mathbf{X})$ denote the $(1-\alpha)$ th posterior quantile of $\theta_{1}$, that is,

$$
P^{\pi}\left[\theta_{1} \leq \theta_{1}^{1-\alpha}(\pi ; \mathbf{X}) \mid \mathbf{X}\right]=1-\alpha,
$$

where $\boldsymbol{\theta}=\left(\theta_{1}, \cdots, \theta_{t}\right)^{T}$ and $\theta_{1}$ is the parameter of interest. We want to find priors $\pi$ for which

$$
P_{\theta}\left[\theta_{1} \leq \theta_{1}^{1-\alpha}(\pi ; \mathbf{X})\right]=1-\alpha+o\left(n^{-r}\right) .
$$

for some $r>0$, as $n$ goes to infinity. Priors $\pi$ satisfying (2.3) are called matching priors. If $r=1 / 2$, then $\pi$ is referred to as a first order matching prior, while if $r=1, \pi$ is referred to as a second order matching prior.

Firstly, we develop the matching prior for the scale parameter. In order to find such matching priors $\pi$, let

$$
\theta_{1}=\alpha \text { and } \theta_{2}=\beta \text {. }
$$


With this parametrization, the likelihood function of parameters $\left(\theta_{1}, \theta_{2}\right)$ for the model $(2.1)$ is given by

$$
L\left(\theta_{1}, \theta_{2}\right)=\frac{\theta_{2} \theta_{1}^{\theta_{2}} x^{\theta_{2}-1}}{\left(\theta_{1}^{\theta_{2}}+x^{\theta_{2}}\right)^{2}} \propto \frac{\theta_{2} \theta_{1}^{\theta_{2}} x^{\theta_{2}}}{\left(\theta_{1}^{\theta_{2}}+x^{\theta_{2}}\right)^{2}} .
$$

Based on (2.4), the Fisher information matrix is given by

$$
\mathbf{I}\left(\theta_{1}, \theta_{2}\right)=\left(\begin{array}{cc}
\frac{\theta_{2}^{2}}{3 \theta_{1}^{2}} & 0 \\
0 & \frac{3+\pi^{2}}{9 \theta_{2}^{2}}
\end{array}\right)
$$

From the above Fisher information matrix $\mathbf{I}, \theta_{1}$ is orthogonal to $\theta_{2}$ in the sense of Cox and Reid (1987). Following Tibshirani (1989), the class of first order probability matching prior is characterized by

$$
\pi_{m}^{(1)}\left(\theta_{1}, \theta_{2}\right) \propto \theta_{1}^{-1} \theta_{2} d\left(\theta_{2}\right)
$$

where $d\left(\theta_{2}\right)>0$ is an arbitrary function differentiable in its argument.

The class of prior given in (2.6) can be narrowed down to the second order probability matching priors as given in Mukerjee and Ghosh (1997). A second order probability matching prior is of the form (2.6), and $d$ must satisfy an additional differential equation (2.10) of Mukerjee and Ghosh (1997), namely

$$
\frac{1}{6} d\left(\theta_{2}\right) \frac{\partial}{\partial \theta_{1}}\left\{I_{11}^{-\frac{3}{2}} L_{1,1,1}\right\}+\frac{\partial}{\partial \theta_{2}}\left\{I_{11}^{-\frac{1}{2}} L_{112} I^{22} d\left(\theta_{2}\right)\right\}=0
$$

where

$L_{1,1,1}=E\left[\left(\frac{\partial \log L}{\partial \theta_{1}}\right)^{3}\right]=0, L_{112}=E\left[\frac{\partial^{3} \log L}{\partial \theta_{1}^{2} \partial \theta_{2}}\right]=-\frac{\theta_{2}}{2 \theta_{1}^{2}}, I_{11}=\frac{\theta_{2}^{2}}{2 \theta_{1}^{2}}, I^{22}=\frac{9 \theta_{2}^{2}}{3+\pi^{2}}$.

Then (2.7) simplifies to

$$
\frac{\partial}{\partial \theta_{2}}\left\{\frac{9 \sqrt{3}}{2\left(3+\pi^{2}\right)} \theta_{1}^{-1} \theta_{2}^{2} d\left(\theta_{2}\right)\right\}=0
$$

Hence the set of solution of (2.8) is of the form $d\left(\theta_{2}\right)=\theta_{2}^{-2}$. Thus the resulting second order probability matching prior is

$$
\pi_{m}^{(2)}\left(\theta_{1}, \theta_{2}\right) \propto \theta_{1}^{-1} \theta_{2}^{-1}
$$

Remark 2.1 There are alternative ways through which matching can be accomplished. Datta et al. (2000) provided a theorem which establishes the equivalence of second order matching priors and HPD matching priors (DiCiccio and Stern, 1994; Ghosh and Mukerjee, 1995) within the class of first order matching priors. The equivalence condition is that $I_{11}^{-3 / 2} L_{111}$ dose not depend on $\theta_{1}$. Since

$$
L_{111}=E\left[\frac{\partial^{3} \log L}{\partial \theta_{1}^{3}}\right]=\frac{\theta_{2}^{2}}{\theta_{1}^{3}},
$$


$I_{11}^{-3 / 2} L_{111}$ does not depend on $\theta_{1}$. Therefore the second order probability matching prior (2.9) is a HPD matching prior. Also

$$
L_{11,1}=E\left[\frac{\partial^{2} \log L}{\partial \theta_{1}^{2}} \frac{\partial \log L}{\partial \theta_{1}}\right]=-\frac{\theta_{2}^{2}}{2 \theta_{1}^{3}}, L_{11,2}=E\left[\frac{\partial^{2} \log L}{\partial \theta_{1}^{2}} \frac{\partial \log L}{\partial \theta_{2}}\right]=-\frac{\theta_{2}}{6 \theta_{1}^{2}}
$$

and $d\left(\theta_{2}\right)=\theta_{2}^{-2}$. Then

$$
\begin{aligned}
& \frac{\partial}{\partial \theta_{2}}\left\{L_{112} I^{22} I_{11}^{-1 / 2} d\left(\theta_{2}\right)\right\}=0, \frac{\partial}{\partial \theta_{2}}\left\{L_{11,2} I^{22} I_{11}^{-1 / 2} d\left(\theta_{2}\right)\right\}=0, \\
& \frac{\partial}{\partial \theta_{1}}\left\{I_{11}^{-3 / 2} L_{111}\right\}=0, \frac{\partial}{\partial \theta_{1}}\left\{I_{11}^{-3 / 2} L_{11,1}\right\}=0 .
\end{aligned}
$$

Therefore the second order matching prior (2.9) matches the alternative coverage probabilities (Mukerjee and Reid, 1999).

Next we develop the matching prior for the shape parameter. Let

$$
\theta_{3}=\beta \text { and } \theta_{4}=\alpha .
$$

Then the Fisher information matrix from (2.5) is given by

$$
\mathbf{I}\left(\theta_{3}, \theta_{4}\right)=\left(\begin{array}{cc}
\frac{3+\pi^{2}}{9 \theta_{3}^{2}} & 0 \\
0 & \frac{\theta_{3}^{2}}{2 \theta_{4}^{2}}
\end{array}\right) .
$$

From the above Fisher information matrix I, the class of first order probability matching prior is characterized by

$$
\pi_{m}^{(1)}\left(\theta_{3}, \theta_{4}\right) \propto \theta_{3}^{-1} d\left(\theta_{4}\right),
$$

where $d\left(\theta_{4}\right)>0$ is an arbitrary function differentiable in its argument. A second order probability matching prior is of the form (2.11), and $d$ must satisfy the following differential equation

$$
\frac{1}{6} d\left(\theta_{4}\right) \frac{\partial}{\partial \theta_{3}}\left\{I_{11}^{-\frac{3}{2}} L_{1,1,1}\right\}+\frac{\partial}{\partial \theta_{4}}\left\{I_{11}^{-\frac{1}{2}} L_{112} I^{22} d\left(\theta_{4}\right)\right\}=0,
$$

where

$$
L_{1,1,1}=E\left[\left(\frac{\partial \log L}{\partial \theta_{3}}\right)^{3}\right]=-\frac{\pi^{2}}{\theta_{3}^{2}}, L_{112}=E\left[\frac{\partial^{3} \log L}{\partial \theta_{3}^{2} \partial \theta_{4}}\right]=0, I_{11}=\frac{3+\pi^{2}}{9 \theta_{3}^{2}} I^{22}=\frac{\theta_{3}^{2}}{3 \theta_{4}^{2}} .
$$

Then (2.12) simplifies to

$$
\frac{1}{6} d\left(\theta_{4}\right) \frac{\partial}{\partial \theta_{3}}\left\{9 \pi^{2}\left(3+\pi^{2}\right)^{-\frac{3}{2}}\right\}=0
$$

Thus the resulting second order probability matching prior is

$$
\pi_{m}^{(2)}\left(\theta_{3}, \theta_{4}\right) \propto \theta_{3}^{-1} d\left(\theta_{4}\right) .
$$


Remark 2.2 Since

$$
L_{111}=E\left[\frac{\partial^{3} \log L}{\partial \theta_{3}^{3}}\right]=\frac{6+\pi^{2}}{6 \theta_{3}^{3}},
$$

$I_{11}^{-3 / 2} L_{111}$ does not depend on $\theta_{3}$. Therefore the second order probability matching prior (2.9) is a HPD matching prior. Also

$$
L_{11,1}=E\left[\frac{\partial^{2} \log L}{\partial \theta_{3}^{2}} \frac{\partial \log L}{\partial \theta_{3}}\right]=\frac{-6+\pi^{2}}{18 \theta_{3}^{3}}, L_{11,2}=E\left[\frac{\partial^{2} \log L}{\partial \theta_{3}^{4}} \frac{\partial \log L}{\partial \theta_{4}}\right]=0 .
$$

Then

$$
\begin{aligned}
& \frac{\partial}{\partial \theta_{4}}\left\{L_{112} I^{22} I_{11}^{-1 / 2} d\left(\theta_{3}\right)\right\}=0, \frac{\partial}{\partial \theta_{4}}\left\{L_{11,2} I^{22} I_{11}^{-1 / 2} d\left(\theta_{4}\right)\right\}=0, \\
& \frac{\partial}{\partial \theta_{3}}\left\{I_{11}^{-3 / 2} L_{111}\right\}=0, \frac{\partial}{\partial \theta_{3}}\left\{I_{11}^{-3 / 2} L_{11,1}\right\}=0 .
\end{aligned}
$$

Therefore the second order matching prior (2.14) matches the alternative coverage probabilities (Mukerjee and Reid, 1999).

\subsection{The reference priors}

Reference priors introduced by Bernardo (1979), and extended further by Berger and Bernardo (1992) have become very popular over the years for the development of noninformative priors. In this section, we derive the reference priors for different groups of ordering of $(\alpha, \beta)$. Then due to the orthogonality of the parameters, following Datta and Ghosh (1995), choosing rectangular compacts for each $\alpha$ and $\beta$ when $\alpha$ or $\beta$ is the parameter of interest, the reference priors are given by as follows.

For the likelihood (2.4), if $\alpha$ or $\beta$ is the parameter of interest, then the reference prior distributions for group of ordering of $\{(\alpha, \beta)\}$ is

$$
\pi_{J}(\alpha, \beta) \propto \alpha^{-1}
$$

For groups of ordering of $\{\alpha, \beta\}$ and $\{\beta, \alpha\}$, the reference prior is

$$
\pi_{R}(\alpha, \beta) \propto \alpha^{-1} \beta^{-1}
$$

Remark 2.3 From the above reference priors, we know that the one-at-a-time reference prior $\pi_{R}$ is the second order matching prior. But Jeffreys' prior $\pi_{J}$ does not satisfy the second order matching criterion.

\section{Implementation of the Bayesian procedure}

We investigate the propriety of posteriors for a general class of priors which includes the reference priors and the matching priors. We consider the class of priors

$$
\pi(\alpha, \beta) \propto \alpha^{-1} \beta^{-a}
$$

where $a \geq 0$. The following general theorem can be proved. 
Theorem 3.1 The posterior distribution of $(\alpha, \beta)$ under the prior (3.1) is proper if $n-a>0$.

Proof: Note that the joint posterior for $\alpha$ and $\beta$ given $\mathbf{x}$ is given by

$$
\pi(\alpha, \beta \mid \mathbf{x}) \propto \alpha^{-1} \beta^{n-a} \prod_{i=1}^{n}\left(\frac{x_{i}}{\alpha}\right)^{\beta}\left[1+\left(\frac{x_{i}}{\alpha}\right)^{\beta}\right]^{-2}
$$

Let $f_{i 1}(\alpha, \beta)=\left(\frac{x_{i}}{\alpha}\right)^{\beta}\left[1+\left(\frac{x_{i}}{\alpha}\right)^{\beta}\right]^{-2}, i=1, \cdots, n$ and $f_{2}(\alpha, \beta)=\left(\frac{x_{\min }}{\alpha}\right)^{\beta}\left[1+\left(\frac{x_{\max }}{\alpha}\right)^{\beta}\right]^{-2}$. Here $x_{\text {min }}=\min \left(x_{1}, \cdots, x_{n}\right)$ and $x_{\text {max }}=\max \left(x_{1}, \cdots, x_{n}\right)$. Then $0<f_{2}(\alpha, \beta)<f_{i 1}(\alpha, \beta)<1$ for $0<\alpha<\infty$ and $0<\beta<\infty$. So there exists a constant $k$ such that $0<f_{i 1} \leq k f_{2}$, $i=1, \cdots, n$. Thus we get

$$
\pi(\alpha, \beta \mid \mathbf{x}) \leq k^{n} \alpha^{-1} \beta^{n-a}\left(\frac{x_{\min }}{\alpha}\right)^{n \beta}\left[1+\left(\frac{x_{\max }}{\alpha}\right)^{\beta}\right]^{-2 n} .
$$

Thus

$$
\int_{0}^{\infty} \int_{0}^{\infty} \pi(\alpha, \beta \mid \mathbf{x}) d \alpha d \beta \leq \int_{0}^{\infty} k^{n} \frac{\Gamma[n] \Gamma[n]}{\Gamma[2 n]} \beta^{n-a-1}\left(\frac{x_{\min }}{x_{\max }}\right)^{n \beta} d \beta
$$

Therefore the last integral (3.4) is finite if $n-a>0$. This completes the proof.

Theorem 3.2 Under the prior (3.1), the marginal posterior density of $\alpha$ is given by

$$
\pi(\alpha \mid \mathbf{x}) \propto \int_{0}^{\infty} \alpha^{-1} \beta^{n-b} \prod_{i=1}^{n}\left(\frac{x_{i}}{\alpha}\right)^{\beta}\left[1+\left(\frac{x_{i}}{\alpha}\right)^{\beta}\right]^{-2} d \beta
$$

and the marginal posterior density of $\beta$ is given by

$$
\pi(\beta \mid \mathbf{x}) \propto \int_{0}^{\infty} \alpha^{-1} \beta^{n-b} \prod_{i=1}^{n}\left(\frac{x_{i}}{\alpha}\right)^{\beta}\left[1+\left(\frac{x_{i}}{\alpha}\right)^{\beta}\right]^{-2} d \alpha .
$$

Note that actually the marginal densities of $\alpha$ and $\beta$ require a one dimensional integration. Therefore, we have the marginal posterior densities of $\alpha$ and $\beta$, and so it is easy to compute the marginal moments of $\alpha$ and $\beta$. In Section 4 , we investigate the frequentist coverage probabilities for Jeffreys prior $\pi_{J}$ and the reference prior $\pi_{r}$, respectively .

\section{Numerical studies}

\subsection{Simulation study}

We evaluate the frequentist coverage probability by investigating the credible interval of the marginal posterior density of $\alpha$ and $\beta$ under the reference prior and Jeffreys' prior given in Section 3 for several configurations $(\alpha, \beta)$ and $n$. That is to say, the frequentist coverage of a $(1-\eta)$ th posterior quantile should be close to $1-\eta$. This is done numerically. Tables 4.1 and 4.2 give numerical values of the frequentist coverage probabilities of $0.05(0.95)$ posterior quantiles for the our prior. The computation of these numerical values is based on the following algorithm for any fixed true $(\alpha, \beta)$ and any prespecified probability value $\eta$. Here $\eta$ is $0.05(0.95)$. Let $\alpha^{\eta}(\pi ; \mathbf{X})$ be the $\eta$ th posterior quantile of $\alpha$ given $\mathbf{X}$. That 
is, $F\left(\alpha^{\eta}(\pi ; \mathbf{X}) \mid \mathbf{X}\right)=\eta$, where $F(\cdot \mid \mathbf{X})$ is the marginal posterior distribution of $\alpha$. Then the frequentist coverage probability of this one sided credible interval of $\alpha$ is

$$
P_{(\alpha, \beta)}(\eta ; \alpha)=P_{(\alpha, \beta)}\left(0<\alpha \leq \alpha^{\eta}(\pi ; \mathbf{X})\right) .
$$

The computed $P_{(\alpha, \beta)}(\eta ; \alpha)$ and $P_{(\alpha, \beta)}(\eta ; \beta)$ when $\eta=0.05(0.95)$ is shown in Table 4.1 and Table 4.2 , respectively. In particular, for fixed $n$ and $(\alpha, \beta)$, we take 10,000 independent random samples of $\mathbf{X}=\left(X_{1}, \cdots, X_{n}\right)$ from the log-logistic distribution.

Table 4.1 Frequentist coverage probability of $0.05(0.95)$ posterior quantiles of $\alpha$

\begin{tabular}{|c|c|c|c|c|}
\hline$\alpha$ & $\beta$ & $n$ & $\pi_{J}$ & $\pi_{r}$ \\
\hline \multirow{9}{*}{0.5} & \multirow{3}{*}{1.0} & 5 & $0.073(0.930)$ & $0.048(0.953)$ \\
\hline & & 10 & $0.061(0.939)$ & $0.051(0.948)$ \\
\hline & & 20 & $0.052(0.945)$ & $0.047(0.950)$ \\
\hline & \multirow{3}{*}{3.0} & 5 & $0.071(0.930)$ & $0.048(0.952)$ \\
\hline & & 10 & $0.060(0.939)$ & $0.052(0.950)$ \\
\hline & & 20 & $0.054(0.943)$ & $0.049(0.948)$ \\
\hline & \multirow{3}{*}{5.0} & 5 & $0.064(0.934)$ & $0.043(0.955)$ \\
\hline & & 10 & $0.056(0.944)$ & $0.047(0.953)$ \\
\hline & & 20 & $0.052(0.950)$ & $0.049(0.953)$ \\
\hline \multirow{9}{*}{1.0} & \multirow{3}{*}{1.0} & 5 & $0.070(0.923)$ & $0.046(0.945)$ \\
\hline & & 10 & $0.058(0.938)$ & $0.047(0.947)$ \\
\hline & & 20 & $0.055(0.945)$ & $0.050(0.950)$ \\
\hline & \multirow{3}{*}{3.0} & 5 & $0.072(0.931)$ & $0.053(0.952)$ \\
\hline & & 10 & $0.059(0.940)$ & $0.049(0.951)$ \\
\hline & & 20 & $0.055(0.950)$ & $0.051(0.955)$ \\
\hline & \multirow{3}{*}{5.0} & 5 & $0.065(0.937)$ & $0.043(0.957)$ \\
\hline & & 10 & $0.058(0.943)$ & $0.049(0.952)$ \\
\hline & & 20 & $0.055(0.946)$ & $0.050(0.950)$ \\
\hline \multirow{9}{*}{5.0} & \multirow{3}{*}{1.0} & 5 & $0.070(0.930)$ & $0.046(0.953)$ \\
\hline & & 10 & $0.060(0.939)$ & $0.051(0.948)$ \\
\hline & & 20 & $0.054(0.941)$ & $0.049(0.947)$ \\
\hline & \multirow{3}{*}{3.0} & 5 & $0.069(0.927)$ & $0.047(0.950)$ \\
\hline & & 10 & $0.059(0.938)$ & $0.048(0.948)$ \\
\hline & & 20 & $0.052(0.944)$ & $0.047(0.948)$ \\
\hline & \multirow{3}{*}{5.0} & 5 & $0.069(0.940)$ & $0.048(0.957)$ \\
\hline & & 10 & $0.056(0.939)$ & $0.046(0.949)$ \\
\hline & & 20 & $0.053(0.947)$ & $0.050(0.952)$ \\
\hline
\end{tabular}

Table 4.2 Frequentist coverage probability of 0.05 (0.95) posterior quantiles of $\beta$

\begin{tabular}{|c|c|c|c|c|}
\hline$\beta$ & $\alpha$ & $n$ & $\pi_{J}$ & $\pi_{r}$ \\
\hline \multirow{9}{*}{1.0} & \multirow{3}{*}{0.5} & 5 & $0.114(0.980)$ & $0.048(0.956)$ \\
\hline & & 10 & $0.089(0.973)$ & $0.048(0.953)$ \\
\hline & & 20 & $0.076(0.965)$ & $0.050(0.950)$ \\
\hline & \multirow{3}{*}{1.0} & 5 & $0.115(0.979)$ & $0.050(0.953)$ \\
\hline & & 10 & $0.094(0.969)$ & $0.051(0.950)$ \\
\hline & & 20 & $0.077(0.965)$ & $0.051(0.949)$ \\
\hline & \multirow{3}{*}{5.0} & 5 & $0.122(0.978)$ & $0.050(0.951)$ \\
\hline & & 10 & $0.093(0.973)$ & $0.054(0.954)$ \\
\hline & & 20 & $0.073(0.969)$ & $0.049(0.952)$ \\
\hline \multirow{9}{*}{3.0} & \multirow{3}{*}{0.5} & 5 & $0.121(0.977)$ & $0.050(0.952)$ \\
\hline & & 10 & $0.091(0.968)$ & $0.050(0.947)$ \\
\hline & & 20 & $0.073(0.968)$ & $0.049(0.950)$ \\
\hline & \multirow{3}{*}{1.0} & 5 & $0.117(0.977)$ & $0.045(0.951)$ \\
\hline & & 10 & $0.087(0.969)$ & $0.049(0.948)$ \\
\hline & & 20 & $0.075(0.968)$ & $0.050(0.953)$ \\
\hline & \multirow{3}{*}{5.0} & 5 & $0.123(0.980)$ & $0.050(0.952)$ \\
\hline & & 10 & $0.085(0.971)$ & $0.049(0.950)$ \\
\hline & & 20 & $0.074(0.964)$ & $0.051(0.949)$ \\
\hline \multirow{9}{*}{5.0} & \multirow{3}{*}{0.5} & 5 & $0.110(0.979)$ & $0.044(0.954)$ \\
\hline & & 10 & $0.086(0.971)$ & $0.049(0.950)$ \\
\hline & & 20 & $0.076(0.968)$ & $0.050(0.952)$ \\
\hline & \multirow{3}{*}{1.0} & 5 & $0.114(0.978)$ & $0.045(0.952)$ \\
\hline & & 10 & $0.091(0.971)$ & $0.051(0.950)$ \\
\hline & & 20 & $0.075(0.967)$ & $0.050(0.952)$ \\
\hline & \multirow{3}{*}{5.0} & 5 & $0.113(0.977)$ & $0.043(0.948)$ \\
\hline & & 10 & $0.092(0.969)$ & $0.050(0.948)$ \\
\hline & & 20 & $0.073(0.966)$ & $0.047(0.949)$ \\
\hline
\end{tabular}


Tables 4.1 and 4.2 indicate that the reference prior meets the target coverage probabilities better than Jeffreys' prior. We also note that the reference prior provides good coverage in small sample size, and the results are less sensitive to the change of the values of $(\alpha, \beta)$. Thus, we recommend to use the reference prior.

\subsection{Real data analysis}

This example is taken from Dey and Kundu (2010). The data is obtained from Lawless (1982), and it represents the number of revolution before failure of each 23 ball bearings in the life tests and they are as follows: $17.88,28.92,33.0,41.52,42.12,45.60,48.8,51.84$, $51.96,54.12,55.56,67.8,68.44,68.64,68.88,84.12,93.12,98.64,105.12,105.84,127.92$, $128.04,173.4$.

Dey and Kundu (2010) concluded that the log-normal distribution and the log-logistic distribution have good fit for this data in terms of the log-likelihood values, KolmogorovSmirnov distances and the $\chi^{2}$ values.

For this data, the maximum likelihood estimates (MLEs) and the corresponding 95\% asymptotic confidence intervals are given in Table 4.3. Also Bayes estimates and the 95\% credible intervals based on the reference prior and Jeffreys' prior given in Table 4.3.

The estimated values of $\alpha$ by MLE, Jeffreys' prior and reference prior are 64.0109, 63.7023 and 63.6226, respectively. And, the estimated values of $\beta$ are 3.3490, 3.3677 and 3.2664. Conclusively, the estimates based on Jeffreys' prior, the reference prior and the MLE give almost same results. The lengths of confidence interval for $\alpha$ based on MLE, Jeffreys' prior and reference prior are 27.3378, 30.7092 and 31.6065. And the lengths of confidence interval for $\beta$ are 2.2642, 2.271 and 2.2495. But for the parameter $\alpha$, the confidence interval based on the MLE is slightly shorter than the credible intervals of the reference prior and Jeffreys' prior.

Table 4.3 Estimate and confidence interval for $\alpha$ and $\beta$

\begin{tabular}{cccc}
\hline \hline Parameter & MLE & $\pi_{J}$ & $\pi_{r}$ \\
\hline$\alpha$ & $64.0109(50.3420,77.6798)$ & $63.7023(49.1155,79.8247)$ & $63.6226(48.6298,80.2363)$ \\
$\beta$ & $3.3490(2.2169,4.4811)$ & $3.3677(2.3157,4.5937)$ & $3.2664(2.2285,4.4780)$ \\
\hline
\end{tabular}

\section{Concluding remarks}

In the log-logistic models, we have found the second order matching prior and the reference prior for both parameters. For the both parameters, we revealed the relevance of the second order matching prior and other matching criteria. We also presented that the reference prior satisfies a second order matching criterion for both parameters. As illustrated in our numerical study, the reference prior seems to be the best appropriate results than Jeffreys' prior in the sense of asymptotic frequentist coverage property. Thus we recommend the use of the reference prior for Bayesian inference of the parameters in the log-logistic distribution.

\section{References}

Ahmad, M. I., Sinclair, C. D. and Werritty, A. (1988). Log-logistic flood frequency analysis. Journal of Hydrology, 98, 205-212.

Bennett, S. (1983). Log-logistic regression models for survival data. Journal of Royal Statistical Society $C$, 32, 165-171. 
Berger, J. O. and Bernardo, J. M. (1989). Estimating a product of means : Bayesian analysis with reference priors. Journal of the American Statistical Association, 84, 200-207.

Berger, J. O. and Bernardo, J. M. (1992). On the development of reference priors (with discussion). In Bayesian Statistics IV, edited by J. M. Bernardo, et al., Oxford University Press, Oxford, 35-60.

Bernardo, J. M. (1979). Reference posterior distributions for Bayesian inference (with discussion). Journal of Royal Statistical Society B, 41, 113-147.

Cox, D. R. and Reid, N. (1987). Orthogonal parameters and approximate conditional inference (with discussion). Journal of Royal Statistical Society B, 49, 1-39.

Datta, G. S. and Ghosh, M. (1995). Some remarks on noninformative priors. Journal of the American Statistical Association, 90, 1357-1363.

Datta, G. S. and Ghosh, M. (1996). On the invariance of noninformative priors. The Annal of Statistics, 24, 141-159.

Datta, G. S., Ghosh, M. and Mukerjee, R. (2000). Some new results on probability matching priors. Calcutta Statistical Association Bulletin, 50, 179-192.

Dey, A. K. and Kundu, D. (2010). Discriminating between the log-normal and log-logistic distributions. Communications in Statistics-Theory and Methods, 39, 280-292.

DiCiccio, T. J. and Stern, S. E. (1994). Frequentist and Bayesian Bartlett correction of test statistics based on adjusted profile likelihood. Journal of Royal Statistical Society B, 56, 397-408.

Fisk, P. R. (1961). The graduation of income distributions. Econometrica, 29, 171-185.

Geskus, R. B. (2001). Methods for estimating the AIDS incubation time distribution when data of seroconversion is censored. Statistics in Medicine, 20, 795-812.

Ghosh, J. K. and Mukerjee, R. (1992). Noninformative priors (with discussion). In Bayesian Statistics IV, edited by J. M. Bernardo, et al., Oxford University Press, Oxford, 195-210.

Ghosh, J. K. and Mukerjee, R. (1995). Frequentist validity of highest posterior density regions in the presence of nuisance parameters. Statistics \& Decisions, 13, 131-139.

Lawless, J. F. (1982). Statistical models and methods for lifetime data, John Wiley and Sons, New York.

Kang, S. G. (2011). Noninformative priors for the common mean in log-normal distributions. Journal of the Korean Data $\& 3$ Information Science Society, 22, 1241-1250.

Kang, S. G., Kim, D. H. and Lee, W. D. (2012). Noninformative priors for the ratio of the scale parameters in the half logistic distributions. Journal of the Korean Data \& Information Science Society, 23, 833-841.

Kang, S. G., Kim, D. H. and Lee, W. D. (2013a). Noninformative priors for the shape parameter in the generalized Pareto distribution. Journal of the Korean Data 8 Information Science Society, 24, 171178.

Kang, S. G., Kim, D. H. and Lee, W. D. (2013b). Noninformative priors for the ratio of parameters of two Maxwell distributions. Journal of the Korean Data \& Information Science Society, 24, 643-650.

Kim, D. H., Kang, S. G. and Lee, W. D. (2009). Noninformative priors for Pareto distribution. Journal of the Korean Data \& Information Science Society, 20, 1213-1223.

Mukerjee, R. and Dey, D.K. (1993). Frequentist validity of posterior quantiles in the presence of a nuisance parameter : Higher order asymptotics. Biometrika, 80, 499-505.

Mukerjee, R. and Ghosh, M. (1997). Second order probability matching priors. Biometrika, 84, 970-975.

Mukerjee, R. and Reid, N. (1999). On a property of probability matching priors: Matching the alternative coverage probabilities. Biometrika, 86, 333-340.

Robson, A. and Reed, D. (1999). Statistical procedures for flood frequency estimation. In Flood Estimation Handbook, 3, Institute of Hydrology, Wallingford, UK.

Shoukri, M. M., Mian, I. U. M. and Tracy, C. (1988). Sampling properties of estimators of log-logistic distribution with application to Canadian precipitation data. Canadian Journal of Statistics, 16, 223236.

Stein, C. (1985). On the coverage probability of confidence sets based on a prior distribution. Sequential Methods in Statistics, Banach Center Publications, 16, 485-514.

Tibshirani, R. (1989). Noninformative priors for one parameter of many. Biometrika, 76, 604-608.

Welch, B. L. and Peers, H. W. (1963). On formulae for confidence points based on integrals of weighted likelihood. Journal of Royal Statistical Society B, 25, 318-329. 